\title{
Failure to detect an 8p22-8p23.1 duplication in patients with Kabuki (Niikawa-Kuroki) syndrome
}

Damien Sanlaville*,1,7, David Genevieve ${ }^{1,7}$, Céline Bernardin ${ }^{1}$, Jeanne Amiel ${ }^{1}$, Clarisse Baumann $^{2}$, Marie-Christine de Blois ${ }^{1}$, Valérie Cormier-Daire ${ }^{1}$, Bénédicte Gerard ${ }^{2}$, Marion Gerard $^{3}$, Martine Le Merrer ${ }^{1}$, Philippe Parent ${ }^{4}$, Fabienne Prieur ${ }^{5}$, Marguerite Prieur ${ }^{1}$, Odile Raoul $^{1}$, Annick Toutain ${ }^{6}$, Alain Verloes ${ }^{1}$, Géraldine Viot ${ }^{1}$, Serge Romana ${ }^{1}$, Arnold Munnich $^{1}$, Stanislas Lyonnet ${ }^{1}$, Michel Vekemans ${ }^{1}$ and Catherine Turleau ${ }^{1}$

${ }^{1}$ Département de Génétique, Hôpital Necker Enfants Malades, Paris, France; ${ }^{2}$ Service de Génétique, Hôpital R Debré, Paris, France; ${ }^{3}$ Service de Génétique, Hôpital Henri Mondor, Créteil, France; ${ }^{4}$ Service de Génétique, CHU de Brest, France; ${ }^{5}$ Service de Génétique, Hôpital Nord, CHU de Saint Etienne, France; ${ }^{6}$ Service de Génétique, Hôpital Bretonneau, CHU de Tours, France

Kabuki syndrome (KS) is a rare MCA/MR syndrome with an estimated frequency of 1/32000 in Japan. This syndrome is characterized by postnatal growth retardation, distinctive facial features, dermatoglyphic anomalies, skeletal dysplasia, and mental retardation. The molecular basis of KS remains unknown. Recently, Milunsky and Huang reported on six unrelated patients with a clinical diagnosis of KS and an 8p22-8p23.1 duplication using comparative genomic hybridization and BAC-FISH studies. Also, they suggested that a paracentric inversion may contribute to the occurrence of KS. In the present study, 24 patients with a clinical diagnosis of KS based on Niikawa-Kuroki criteria have been collected. They were tested for the presence of an $8 p$ duplication using the same clones as described by Milunsky and Huang. Our results do not confirm the previously described association between KS and an 8p22-8p23.1 duplication.

European Journal of Human Genetics (2005) 13, 690-693. doi:10.1038/sj.ejhg.5201383

Published online 16 March 2005

Keywords: Kabuki syndrome; chromosome 8p22-8p23 duplication; $\beta$-defensin gene cluster

\section{Introduction}

Kabuki syndrome (KS, Niikawa-Kuroki syndrome, MIM: 147920) is a rare multiple congenital anomaly/mental retardation syndrome described simultaneously by Niikawa $e t a l^{1}$ and Kuroki et $a .^{2}$ The estimated frequency of this syndrome is about in 1/32000 in Japan. Thus far, more than 300 patients have been described in the literature. ${ }^{3}$ The syndrome is characterized by postnatal growth retardation, distinctive facial features, dermatoglyphic

*Correspondence: Dr D Sanlaville, Hôpital Necker-Enfants Malades, 149 rue de Sèvres, 75743 Paris Cedex 15, France. Tel: + 331444949 48; Fax: + 331444904 17; E-mail: damien.sanlaville@nck.ap-hop-paris.fr ${ }^{7}$ These authors contributed equally to this work

Received 18 August 2004; revised 28 October 2004; accepted 2 December 2004 anomalies with persistence of fetal fingertip pads, skeletal dysplasia, and mental retardation. In addition, a broad spectrum of clinical findings are observed in KS patients such as cardiac and kidney malformations, autoimmune thrombopenia and anemia, growth hormone deficiency and premature thelarche (review in Matsumoto and Niikawa $^{4}$ ).

The syndrome is usually sporadic. An autosomal dominant mode of inheritance has been proposed in KS because of vertical transmission in several families, a nonincreased rate of consanguinity and a normal sex ratio. ${ }^{5}$ The multisystemic nature of $\mathrm{KS}$ suggests that it might be caused by a microdeletion or microduplication affecting several genes or by a single gene that regulates various target genes. 
Recently, Milunsky and Huang ${ }^{6}$ using comparative genomic hybridization and BAC-FISH studies reported six unrelated patients with KS and an 8p22-8p23.1 duplication. The duplicated region is comprised between BAC clones RP11-112G9 and RP11-92C1. It is approximately $3.5 \mathrm{Mb}$ in size. Furthermore, they reported the presence of a paracentric inversion involving BAC RP11-122N11 in all six patients and the two mothers studied. This inversion is not contiguous to the duplication. In one out of 20 controls a larger inversion was found.

\section{Materials and methods}

We collected a series of 24 patients with a clinical diagnosis of KS. They were ascertained through medical genetics units. They met the KS clinical criteria described by Niikawa et $a l^{1}$ and Kuroki et $a l^{2}$ namely postnatal short stature, mental retardation, skeletal anomalies, dermatoglyphic anomalies, and characteristic facial features (Figure 1). The clinical data are presented in Table 1. All patients were born to unrelated parents and are sporadic cases. Cardiac, renal, ophthalmologic, audiometric, and metabolic investigations including plasma aminoacids, as well as urine organic acids chromatography, and a protein

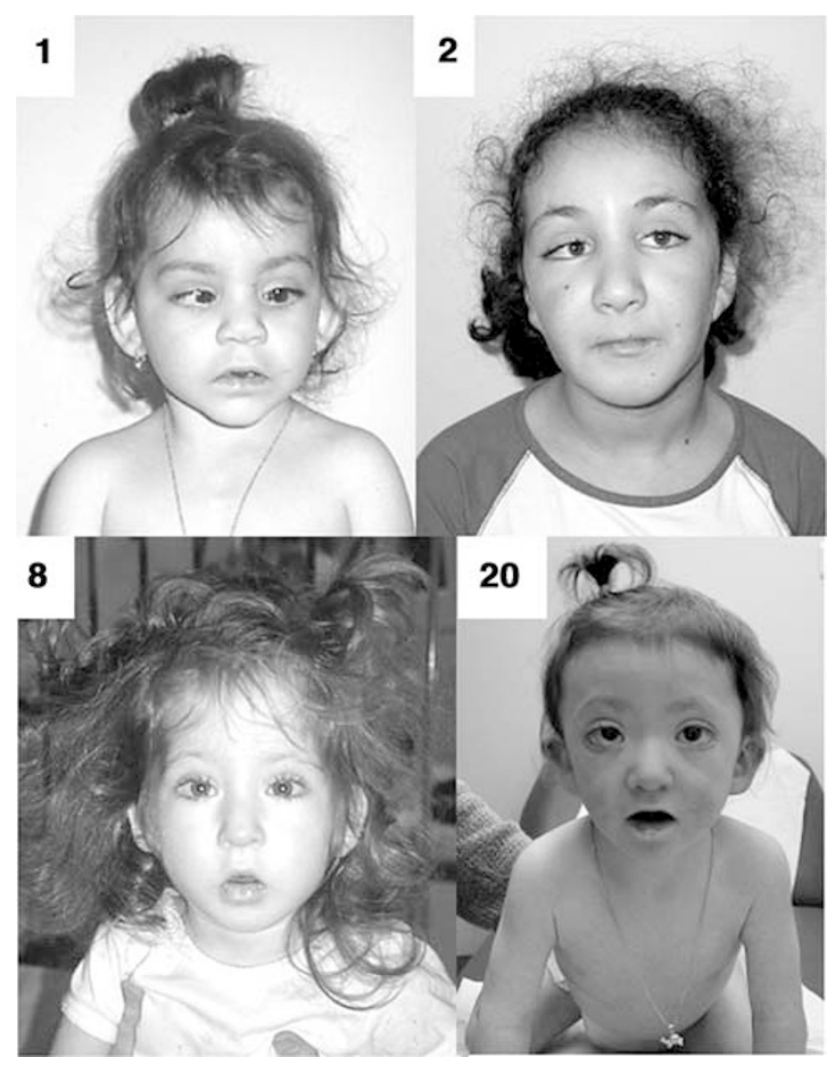

Figure 1 Distinctive facial features for cases 1, 2, 8, and 20. glycosylation test were carried out in all patients. Appropriate ethical approval and written informed consent were obtained. Out of these 24 patients, 10 were published previously (Patients 1, 3, 4, 7, 10, 11, 13, and 14). ${ }^{7-9}$

Standard and high-resolution chromosome analyses were obtained from cultured peripheral blood lymphocytes. FISH experiments were performed as described previously. ${ }^{10}$

All patients were studied with BACs RP11-235F10, RP11252K12, RP11-31B7 to detect the 8p duplication and BAC RP11-122N11 to study the paracentric inversion as described by Milunsky and Huang. ${ }^{6}$ In addition, we used in six patients BACs RP11-11P7, RP11-140K14, RP11-112G9, RP11-92C1, RP11-23H1, and RP11-141K9 that are other clones included in the 8p22-8p23.1 duplication. For each patient we counted 10 metaphases and 10 nuclei.

\section{Results}

All patients had a normal karyotype at a 500-850 bands resolution level. In all KS patients only one signal on each chromosome was observed using BAC RP11-235F10, RP11$252 \mathrm{~K} 12$, and RP11-31B7 on metaphases as well as on interphase nuclei (Figure 2a and b). Also no duplication was detected using BACs RP11-11P7, RP11-140K14, RP11112G9, RP11-92C1, RP11-23H1, and RP11-141K9. Regarding the postulated paracentric inversion, two signals on each homologous chromosome, or two signals on one chromosome and one on the other were observed using BAC RP11-122N11 hybridization on interphase nuclei. Same results were obtained in phenotypically normal controls.

\section{Discussion}

The aim of the present study of KS patients was to determine the frequency of an $8 \mathrm{p} 22-8 \mathrm{p} 23.1$ duplication observed previously by Milunski and Huang. ${ }^{6}$ Contrary to these authors, we were not able to detect any $8 p$ duplication in our series of 24 Caucasians KS selected according to the clinical Niikawa-Kuroki criteria. Similarly, Miyake et $\mathrm{al}^{11}$ failed to find any duplication in 28 Asiatic patients with typical KS.

Several patients with $8 \mathrm{p} 23$ duplication have been reported previously. In most cases this duplication was believed to be a benign variant with no phenotypic effect ${ }^{12}$ and described as an euchromatic variant (EV). ${ }^{13,14}$ This was disputed by Tsai $e t a l^{15}$ who reported on 12 patients with an 8 p23 duplication and a significant phenotype comprising mainly heart defects. Nevertheless, the clinical features of these patients were not evocative of KS. Some of them presented developmental delay, short stature, cardiac anomalies and hypotonia, but no skeletal and dermatoglyphic anomalies nor facial features characteristic of KS 
Table 1 Clinical findings in our $24 \mathrm{KS}$ patients compared to the literature data

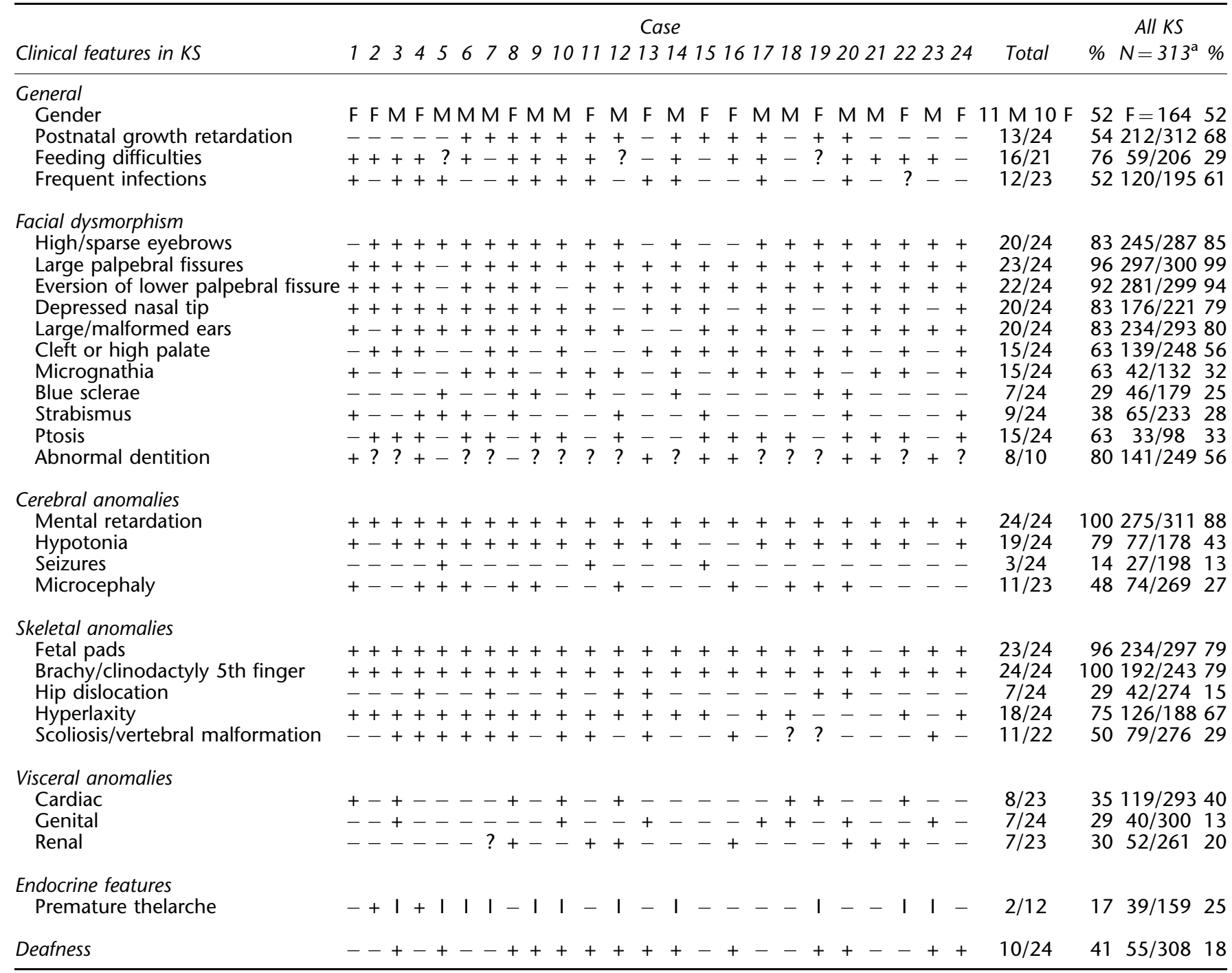

I = Inappropriate; ? = not documented.

${ }^{a}$ The number of patients assessed for each feature are based on the data available in the literature. ${ }^{4}$

were observed. The phenotypic variability could be related to the size of the duplicated segment as well as to the region involved. In fact, the breakpoints have not been characterized at the molecular level in any of these patients. It is tempting to locate the breakpoints within the olfactory repeat region (ORRs) described by Giglio et al. ${ }^{16,17}$ However, it is possible that alternative LCRs can mediate rearrangements with subtle difference in the amount of deleted/duplicated material. ${ }^{18}$

Based on FISH results obtained with the BAC RP11122N11, Milunsky and Huang ${ }^{6}$ suggested that a paracentric inversion may contribute to the occurrence of the $8 \mathrm{p}$ duplication. Using the same probe, Miyake et $a l^{11}$ observed duplicated rather than inverted signals. Among $22 \mathrm{KS}$ patients, they found 15 homozygous and seven hetero- zygous duplications. They observed the same frequency of homozygous and heterozygous duplications in a cohort of 52 normal individuals. Using a combination of multiplex amplifiable probe hybridization and semiquantitative fluorescence in situ hybridization (SQ-FISH), Hollox et al ${ }^{19}$ observed a variation in DNA copy number across band 8p23.1. They showed that a cluster of at least three antimicrobial $\beta$ defensin genes is present at $8 \mathrm{p} 23.1$. The copy number of this cluster is variable. The most frequent copy numbers are three or four. Chromosomes with seven or eight copies of this unit can be identified at the cytogenetic level in patients carrier of the previously described 8p23.1 EV. ${ }^{19}$ Interestingly, BAC RP11-122N11 probe maps precisely in the middle of this $\beta$-defensin-gene cluster and corresponds to SPAG11 gene. This BAC is 

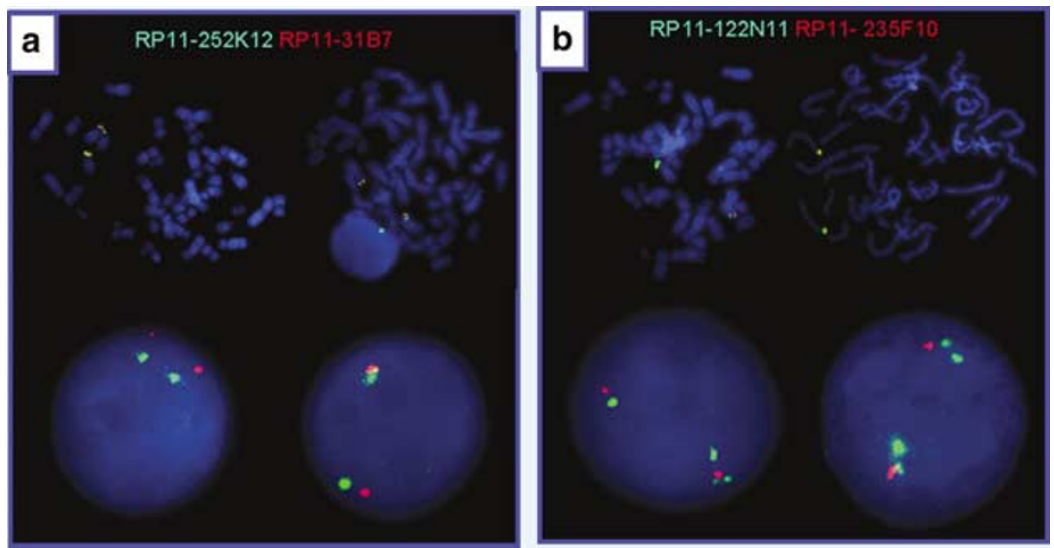

Figure 2 FISH results for cases 1 and 8. (a) BACs RP11-252K12 (green) and RP11-31B7 (red) on metaphases (upper) and interphase nuclei (lower). Only two signals were observed for each probe. These two probes are duplicated in patients reported by Milunsky and Huang. (b) BACs RP11-122N11 (green) and RP11-235F10 (red) on metaphase (upper) and interphase nuclei (lower). Only two signals were observed for the BAC RP11-235F10. The BAC RP11-122N11 may generate two signals on each homologous chromosome (right) or on one chromosome (left). This most likely corresponds to a polymorphism (see text).

estimated to be $10 \mathrm{~kb}$ in length (http://genome.ucsc.edu/ cgi-bin/hgGateway). Two locations in 8p23 (7.3 and $7.8 \mathrm{Mb}$ ) are given in NCBI Clone Registry (http:// www.ncbi.nlm.nih.gov/genome/clone/). So, we would like to suggest that the variable number of signals generated by BAC RP11-122N11 results from a polymorphism linked to the genome architecture of this region rather than a paracentric inversion.

In conclusion, using high-resolution chromosomes and BAC-FISH analysis we failed to detect an 8p22-8p23.1 duplication or a paracentric inversion in a series of $24 \mathrm{KS}$ patients. Further studies, for example using micro-array CGH, might be useful to identify a recurrent chromosomal imbalance in KS.

\section{Acknowledgements}

We are grateful to M Picq and C Estrade for excellent technical support. $B A C$ clones were a gift from Dr Heiling, Genoscope, Evry, France. We thank the children of the Institut Médico-éducatif 'Les Glycines', SaintGermain-en-Laye, France and their parents for their financial help. This work was supported by the PHRC No. AOM 02122.

\section{References}

1 Niikawa N, Matsuura N, Fukushima Y et al: Kabuki make-up syndrome: a syndrome of mental retardation, unusual facies, large and protruding ears, and postnatal growth deficiency. I Pediatr 1981; 99: 565-569.

2 Kuroki Y, Suzuki Y, Chyo H et al: A new malformation syndrome of long palpebral fissures, large ears, depressed nasal tip, and skeletal anomalies associated with postnatal dwarfism and mental retardation. J Pediatr 1981; 99: 570-573.

3 Wessels MW, Brooks AS, Hoogeboom J et al: Kabuki syndrome: a review study of three hundred patients. Clin Dysmorphol 2002; 11: 95-102.

4 Matsumoto N, Niikawa N: Kabuki make-up syndrome: a review. Am J Med Genet 2003; 117C: 57-65.

5 Tsukahara M, Kuroki Y, Imaizumi K et al: Dominant inheritance of Kabuki make-up syndrome. Am J Med Genet 1997; 73: 19-23.
6 Milunsky JM, Huang XL: Unmasking Kabuki syndrome: chromosome 8 p22-8p23.1 duplication revealed by comparative genomic hybridization and BAC-FISH. Clin Genet 2003; 64: 509-516.

7 Toutain A, Plee Y, Ployet MJ et al: Deafness and Mondini dysplasia in Kabuki (Niikawa-Kuroki) syndrome. Report of a case and review of the literature. Genet Couns 1997; 8: 99-105.

8 Kluijt I, van Dorp DB, Kwee ML et al: Kabuki syndrome - report of six cases and review of the literature with emphasis on ocular features. Ophthalmic Genet 2000; 21: 51-61.

9 Genevieve D, Amiel J, Viot G et al: Atypical findings in Kabuki syndrome: report of 8 patients in a series of 20 and review of the literature. Am J Med Genet 2004; 129A: 64-68.

10 Romana SP, Cherif D, Le Coniat M et al: In situ hybridization to interphase nuclei in acute leukemia. Genes Chromosomes Cancer 1993; 8: 98-103.

11 Miyake N, Harada N, Shimokawa O et al: On the reported 8p22-p23.1 duplication in Kabuki make-up syndrome (KMS) and its absence in patients with typical KMS. Am J Med Genet 2004; 128A: $170-172$.

12 Engelen JJ, Moog U, Evers JL et al: Duplication of chromosome region 8p23.1 $\rightarrow$ p23.3: a benign variant? Am J Med Genet 2000; 91: 18-21.

13 Barber JC, Joyce CA, Collinson MN et al: Duplication of 8p23.1: a cytogenetic anomaly with no established clinical significance. J Med Genet 1998; 35: 491-496.

14 O'Malley DP, Storto PD: Confirmation of the chromosome 8p23.1 euchromatic duplication as a variant with no clinical manifestations. Prenat Diagn 1999; 19: 183-184.

15 Tsai CH, Graw SL, McGavran L: 8p23 duplication reconsidered: is it a true euchromatic variant with no clinical manifestation? J Med Genet 2002; 39: 769-774.

16 Giglio S, Broman KW, Matsumoto N et al: Olfactory receptor-gene clusters, genomic-inversion polymorphisms, and common chromosome rearrangements. Am J Hum Genet 2001; 68: 874-883.

17 Giglio S, Calvari V, Gregato G et al: Heterozygous submicroscopic inversions involving olfactory receptor-gene clusters mediate the recurrent t(4;8)(p16;p23) translocation. Am J Hum Genet 2002; 71: $276-285$.

18 Shaw CJ, Withers MA, Lupski JR: Uncommon deletions of the Smith-Magenis syndrome region can be recurrent when alternate low-copy repeats act as homologous recombination substrates. Am J Hum Genet 2004; 75: 75-81.

19 Hollox EJ, Armour JA, Barber JC: Extensive normal copy number variation of a beta-defensin antimicrobial-gene cluster. Am J Hum Genet 2003; 73: 591-600. 